Rev. Elev. Méd. Vét. Pays trop.. 1967, 20, 3 (475-483).

\title{
Utilisation de l'urée pour l'alimentation des veaux au sevrage
}

\author{
por H. SERRES, P. CAPITAINE, J. GILIBERT
}

\begin{abstract}
RÉSUMÉ
A Madagascar, au Centre de Kianjasoa, 66 jeunes bovins ( $3 / 4$ brahma $x$ $1 / 4$ Zébu Malgache) ant été alimentés durant leur sevrage avec une ration à base de foin, donné à volonté, et additionné d'urée.

Les résultats pondéraux ainsi que les bilans nutritionnel et économique sont donnés et discutés.

L'effet favorable de l'urée dans une alimentation des veaux à base de foin s'est vu entièrement confirmé.
\end{abstract}

Les Centres de Recherches Zootechnıques que gère I'I. E.M. V.T. à Madagascar sont situés dans des zones tropicales caractérisées par une longue saison sèche qui dure environ 7 mois d'avril à novembre.

Il a été observé que pour le meilleur élevage des veaux dans des conditions extensives, il était souhaitable qu'ils naissent jusłe avant la salson des pluies, pour que la mère dispose d'une nourriture abondante pendant la lactation, ef pour que le veau dispose d'herbe alibile lorsqu'il atternt 3 mois.

On est donc conduit à effectuer une saison de monte au cours des mols de décembre, janvier, février, qui coïncide d'ailleurs, avec le maximum de fécondité des vaches. De ce fait les naissances ont lieu en septembre, octobre, novembre.

Pour éviter que deux générations de veaux ne soient mélangées, on doit séparer chaque année les veaux de leur mère aux alentours du 1 er août. Les veaux les plus âgés ont 11 mois, les plus jeunes, 8 mois.

Il est nécessaire de les maintenir dans des parcs pour éviter qu'ils ne s'enfuient pour retrouver leur mère. La nourriture qu'on pourra leur distribuer sera forcément médiocre, car on va se trouver au cours de la fin de la saison sèche. Parmi les solutions oblıgatoirement peu onéreuses, l'alimentation au foin a été retenue.

Mais, comme au moment où les graminées pourraient donner un foin de qualité il pleut beaucoup, on est conduit à retarder le fauchage pour permettre un fanage assez facile. II s'en suit que le foin est presque toujours médiocre.

On distribue en supplément $50 \mathrm{~g}$ par jour et par animal d'un mélange qui contient de la farıne d'os calcınés, du sel, et de la vitamıne $A$.

II n'en demeure pas moins qu'une telle alimentation, pour économique qu'elle soit, ne permet pas la crossance normale des animaux.

II est facile de voir qu'elle est assez fortement hypoazotée.

Corriger l'apport azoté par du tourtecu d'arachides constitue une solution onéreuse, et doit mettre en cuvre des quantités telles qu'elle ne représenterait pas une solution vulgarisable.

C'est pourquol, Il a été décıdé de faire appel à l'urée.

Comme le rappelait LADRAT (5) l'influence 
favorable de l'urée dans l'alimentation des ruminants a été observée depuis très longtemps.

Il est aujourd'hui bien connu, depuis les travaux de LOOSLI (6) que l'urée est utilisée par les microorganismes du rumen qui sont capables de faire avec elle la synthèse des acides aminés indispensables. WATSON (7) utilisant l'isotope «15» de l'azote montra que la synthèse allait ainsi jusqu'aux protéines, lesquelles sont utilisées par le ruminant lorsqu'il digère les microorganismes.

De nombreux travaux ont établi l'utılité de l'urée dans les rations d'animaux en croissance. FERRANDO (2) en a donné une étude synthétique.

Nous rappellerons seulement que I'utilisation de l'urée demeure soumise à certaines conditions:

10 Elle doif n'être distribuée qu'à des doses journalières modérées (150 g au maximum pour une vache adulte).

20 Il est préférable qu'elle n'assure qu'une partie de l'apport azoté : 40 p. 100 selon HART et Coll. (3) dans le cas du veau.

3o Une certaine quantıté d'amıdon facilement digestıble permet une meilleure synthèse protéique à partir de l'urée. JARRIGE (4) dans son important travail sur l'utilisation des glucides par les ruminants I'a nettement mis en évidence.

Une expérience prélıminaire effectuée à Miadana (Région de Majunga, Altitude $50 \mathrm{~m}$ ) par
BUCK et Coll. (1) a montré que l'addition de $20 \mathrm{~g}$ par jour et par animal d'urée, à une ration à base de foin et de farine basse de riz, permettait une augmentation significative de la croissance.

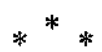

Au cours du sevrage 1965 au Centre de Kianjasoa (Région du Moyen-Ouest, Altitude $900 \mathrm{~m}$ ) une expérience a été réalısée, pour déterminer l'amélioration obtenue dans une alimentation à base de foin «ad libıtum » par addition d'urée ; on a également comparé deux quantités de manioc sec pour l'apport d'une certaine quantité d'amidon. Comme ce manioc n'apporte pratiquement qucune matière azotée digestible, la quantité d'urée distribuée a été portée à $50 \mathrm{~g}$ par anımal et par jour.

\section{MATÉRIEL ET MÉTHODES}

66 jeunes anımaux (3/4 Brahma, 1/4 Zébu malgache) furent utilisés et répartis en six lots de 11 , dont 3 lots de femelles et 3 lots de mâles. Chaque lot étalt maintenu dans un petit parc, avec ratelier à foin, mangeoire et abreuvorr (eau à volonté).

Les aliments utilisés étaient du foin de Brachiaria ruziziensis, récolté tardivement et assez grossier, ainsı que de la farine de manıoc sec et de la poudre d'os, dont les compositions sont rapportées dans le tableau no 1.

TABLEAU I. - Composition des aliments donnés aux vequx

\begin{tabular}{|c|c|c|c|}
\hline & $\mathrm{F} O \mathrm{IN}$ & $M A N I O C$ & OS SECS \\
\hline Humiditē & $9,10 \mathrm{p} \cdot \mathrm{IO0}$ & $12,3 \mathrm{~g} \cdot 100$ & 4 p. 100 \\
\hline Matières minërales & $4,52 \mathrm{p} \cdot 100$ & $I, 3$ p. I00 & \\
\hline Matières grasses & $\mathrm{I}, 82 \mathrm{p} \cdot \mathrm{IOO}$ & $\mathrm{I}, \mathrm{I}$ p. IOO & \\
\hline $\begin{array}{l}\text { Matières azotées brutes } \\
\qquad(\mathrm{N} \times 6,25)\end{array}$ & $5,33 \mathrm{p} \cdot \mathrm{IO0}$ & 2,2 p. I00 & \\
\hline Cellulose brute (Wende) & . $29,80 \mathrm{p} \cdot \mathrm{IOO}$ & $3,-$ p. I00 & \\
\hline Extractif non azoté & $49,43 \mathrm{p} \cdot 100$ & $80, I \mathrm{p} \cdot \mathrm{IOO}$ & \\
\hline Insoluble chlorhydrique & $\mathrm{I}, 48 \mathrm{p} \cdot \mathrm{I} 00$ & 0,3 p. 100 & \\
\hline Phosphore (en P) & $0,072 \mathrm{p} \cdot \mathrm{IOO}$ & $0,029 p .100$ & I6 p. I00 \\
\hline Calcium (en Ca) & $0,263 \mathrm{p} \cdot I 00$ & $0,2 \mathrm{I} 2 \mathrm{p} .100$ & $35 \mathrm{p} .100$ \\
\hline
\end{tabular}


L'urée est à 45 p. 100 d'azote. Pour qu'elle soit consommée, elle doit être mélangée au manioc.

Chaque animal recevait un complément de $20.000 \mathrm{U}$.l. de Vitamine A, car l'alimentation ne comportait aucun fourrage vert ainsi que $10 \mathrm{~g}$ de sel (Tableau II).
L'expérimentation s'est poursuivie pendant 3 mais du 25 août au 26 novembre après une période d'adaptation de 1 mois, au cours de laquelle les animaux étaient tous au même régime (foin + poudre d'os + sel + Vit. A).

TABLEAU II. - Raton alimentase distribuée par animal et par Jour

\begin{tabular}{|c|c|c|c|c|c|c|}
\hline & $\begin{array}{c}\text { LOT I } \\
\text { (femelles) }\end{array}$ & $\begin{array}{l}\text { LOT } 2 \\
\text { (femelles) }\end{array}$ & $\begin{array}{l}\text { LOT } 3 \\
\text { (femelles) }\end{array}$ & $\begin{array}{c}\text { LOT } 4 \\
\text { (mâles) }\end{array}$ & $\begin{array}{l}\text { LOT } 5 \\
\text { (mâles) }\end{array}$ & $\begin{array}{l}\text { LOT } 6 \\
\text { (mâles) }\end{array}$ \\
\hline UREE (g) & - & 50 & 50 & 50 & 50 & - \\
\hline MANIOC (g) & 250 & 250 & I. 250 & 250 & I. 250 & I. 250 \\
\hline FOIN & & Foin distr 1 & à volonté & & & \\
\hline OS CALCINES ( $\mathrm{g}$ ) & 30 & 30 & 30 & 30 & 30 & 30 \\
\hline SEL ( $(B)$ & Io & Io & Io & Io & Io & I0 \\
\hline VITAMINE A (U.I.) & 20.000 & 20.000 & 20.000 & 20.000 & 20.000 & 20.000 \\
\hline
\end{tabular}

\section{RÉSULTATS}

\section{Résultats pondéraux.}

Les animaux ont été pesés individuellement toutes les semaines, et les poids moyens ont été établis chaque semaine par lot.
Les courbes de poids moyens sont figurées aux graphiques 1 à 6 .

Le bilan pondéral de l'expérience est donné dans le tableau III.

TABLEAU III. - Bilan pondéral de l'expérience

\begin{tabular}{|c|c|c|c|c|c|c|}
\hline & LOT I & LOT 2 & LOT 3 & LOT 4 & LOT 5 & LOT 6 \\
\hline $\begin{array}{l}\text { Polds moyens animaux } \\
\text { au } 26 \text { Août } 1965(\mathrm{~kg})\end{array}$ & $I 66,2$ & 164,7 & $1] 2,6$ & 177,4 & I77,2 & 184,6 \\
\hline $\begin{array}{l}\text { Poids moyens animaux } \\
\text { au } 25 \text { Novembre }(\mathrm{Kg})\end{array}$ & $\mathrm{I} 74,0$ & 192,2 & 194,9 & 207,6 & 203,0 & I85, 9 \\
\hline $\begin{array}{l}\text { Crolssance pondërale } \\
\text { moyenne (kg) }\end{array}$ & $+7,8$ & $+27,5$ & $+22,3$ & $+30,2$ & $+25,8$ & $+I, 3$ \\
\hline $\begin{array}{l}\text { Crolssance pondérale } \\
\text { du lot }\left(\mathrm{K}_{8}\right)\end{array}$ & 85,8 & 302,5 & 245,3 & 332,2 & 283,8 & I 4,3 \\
\hline
\end{tabular}

L'étude statistique montre :

a) En ce qui concerne l'influence du sexe.

Les lots 2 (femelles) et 4 (mâles) sont au même régime. Il en est de même pour les lots 3 (fernelles) ef 5 (mâles). Dans chaque cas les mâles ont une croissance légèrement supérieure à celle des femelles, mas cette différence n'est pas significotive. 
b) Influence de l'urée.

Les lots 1 et 2 sont au même régime à l'exception de l'urée qui est administrée au lot 2 et pas au lot 1.

Les animaux du lot 2 ont un gain de poids moyen supérieur de manière hautement significative à celui du lot 1 .

L'urée a donc une utilité incontestable lorsqu'elle est associée d̀ une ration de foin «ad libitum » et de manioc sec ( $250 \mathrm{~g} / \mathrm{jour}$ ).

Bien que le lot 4 soit constitué de mâles, ce qui interdit une comparaison rigoureuse avec le lot 1 , le résultat obtenu vient confirmer l'effet bénéfique de l'urée.

c) Influence d'une distribution abondante de manioc.

Les lots de femelles 2 et 3 ont reçu la même alimentation à l'exception du manıoc : $250 \mathrm{~g} / \mathrm{jour}$ pour le lot 2, $1.250 \mathrm{~g} / \mathrm{jour}$ pour le lot 3 .
La comparaison des moyennes de gains journaliers montre une certaine supériorité de la ration à $250 \mathrm{~g}$ de manioc, mais la différence n'est pas significative.

Pour la même raison, les moyennes de gains journaliers des mâles des lots 4 et 5 sont comparables. On aboutit exactement aux mêmes conclusions.

Il ne parâ̂t pas intéressant d'accroître la quantité de manıc de la ration pour favoriser l'utilisation de l'urée. Nous verrons par la suite combien cela se justifie sur le plan nutritionnel.

d) La distribution de $1.250 \mathrm{~g} / \mathrm{jour}$ de manıo sans urée, conduit à un échec (lot 6)

\section{$2^{\circ}$ Bilan nutritionnel.}

Au cours de l'expérience les consommations ont été les suivantes (Tableau IV).

TABLEAU IV. - Bilan nutritiorel

\begin{tabular}{|c|c|c|c|c|c|c|}
\hline & LOT I & LOT 2 & LOT 3 & LOT 4 & LOT 5 & LOT 6 \\
\hline Foin $(\mathrm{Kg})$ & 5952 & 7036 & 6456 & 7344 & 5976 & 5328 \\
\hline Manioc $(\mathrm{Kg})$ & 2 IO & 2 IO & I2I.8 & 2 I0 & I2I8 & I2I8 \\
\hline Urée $(\mathrm{Kg})$ & 0 & 47 & 47 & 47 & 47 & 0 \\
\hline
\end{tabular}

On constate que les lats $2.3,4,5$, qui ont reçu de l'urée ont consommé davantage de foin que les lots 1 et 6 qui n'en disposaient pas. Il se trouve donc vérifié que l'urée favorise l'utilisation des fourrages grossiers.

Parmi les lots qui recevaient de l'urée, ceux qui consommaient $250 \mathrm{~g}$ de manioc (lots 2 et 4) ont mangé plus de foin que les lots 3 et 6 qui avaient $1.250 \mathrm{~g}$ de manioc.

Les animaux qui ont consommé bequcoup de manioc (qui est toujours apprécié) ont réduit leur consommation de foin.

Si l'on tient compte des compositions des aliments de base données plus haut, ainsi que des données ci-après :

$-1 \mathrm{~kg}$ du foin utilisé représente 0,4 U. F.

$-1 \mathrm{~kg}$ de farine de manioc représente 0,9 U. F.
- L'équivalent protéique de l'urée distribuée (poids $\times 0,45 \times 6,25$ ) est de $131 \mathrm{~kg}$ par lot en ayant reçu.

On pourra calculer les unités fourragères et les matières azotées brutes consom mées et établir un rapport matières azotées brutes/Unités fourragères dans la ration de chaque lot (Tableau V).

La matière azotée digestible ne pourra être qu'appréciée, car la digestibilité du foin utilisé n'est pas connue, et l'utilisation digestive de l'urée a donné lieu à controverses.

Les lots $3,5,6$, qui ont bénéficié du manioc à dose forte sont ceux qui ont consommé au total le plus grand nombre d'U. F.

Si l'on s'en tenait à cela, on devrait les retrouver en tête pour ce qui est de la croissance. Ce n'est pas le cas, et cela s'explique aisément si I'on examine le rapport M. A. B./U. F. 

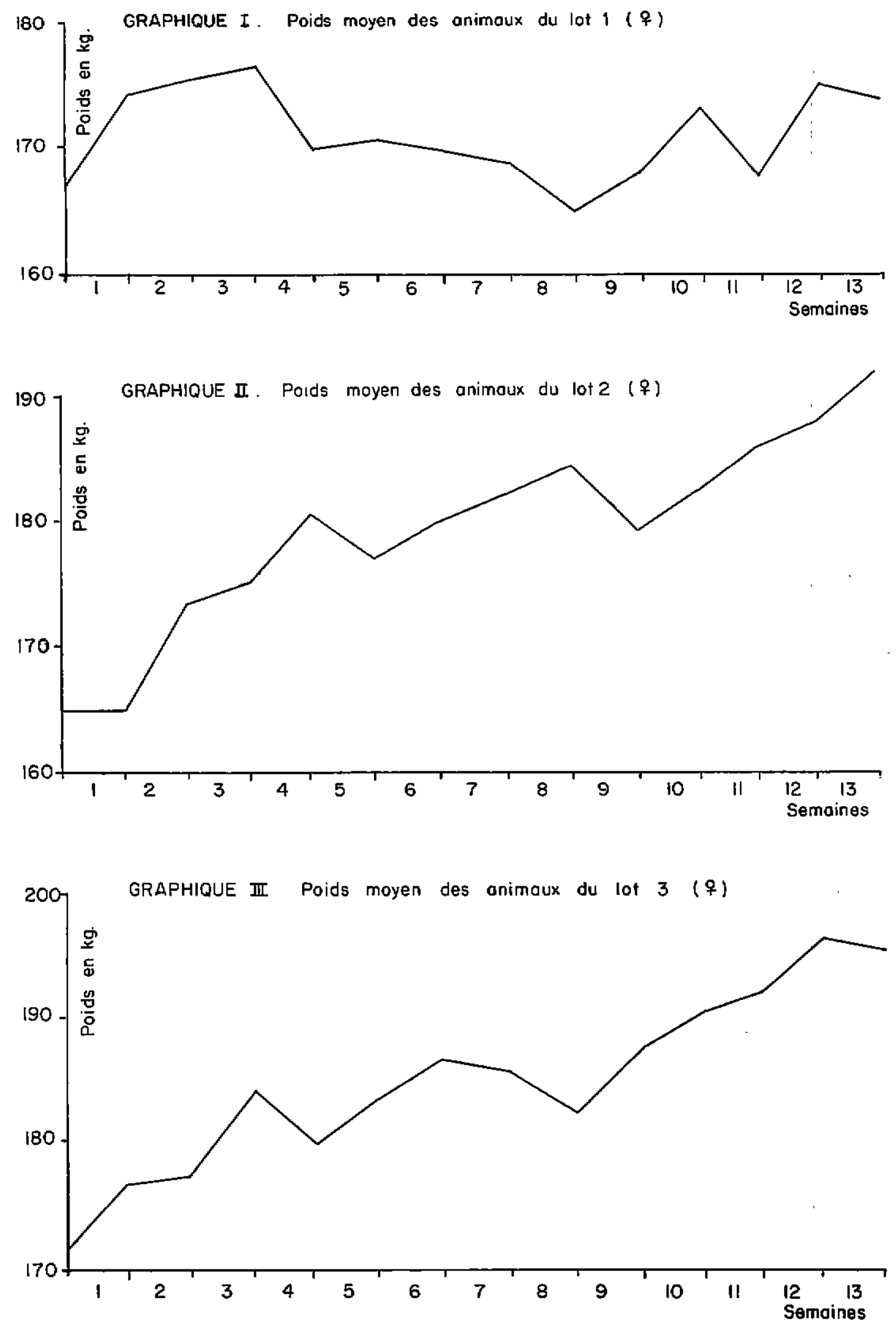

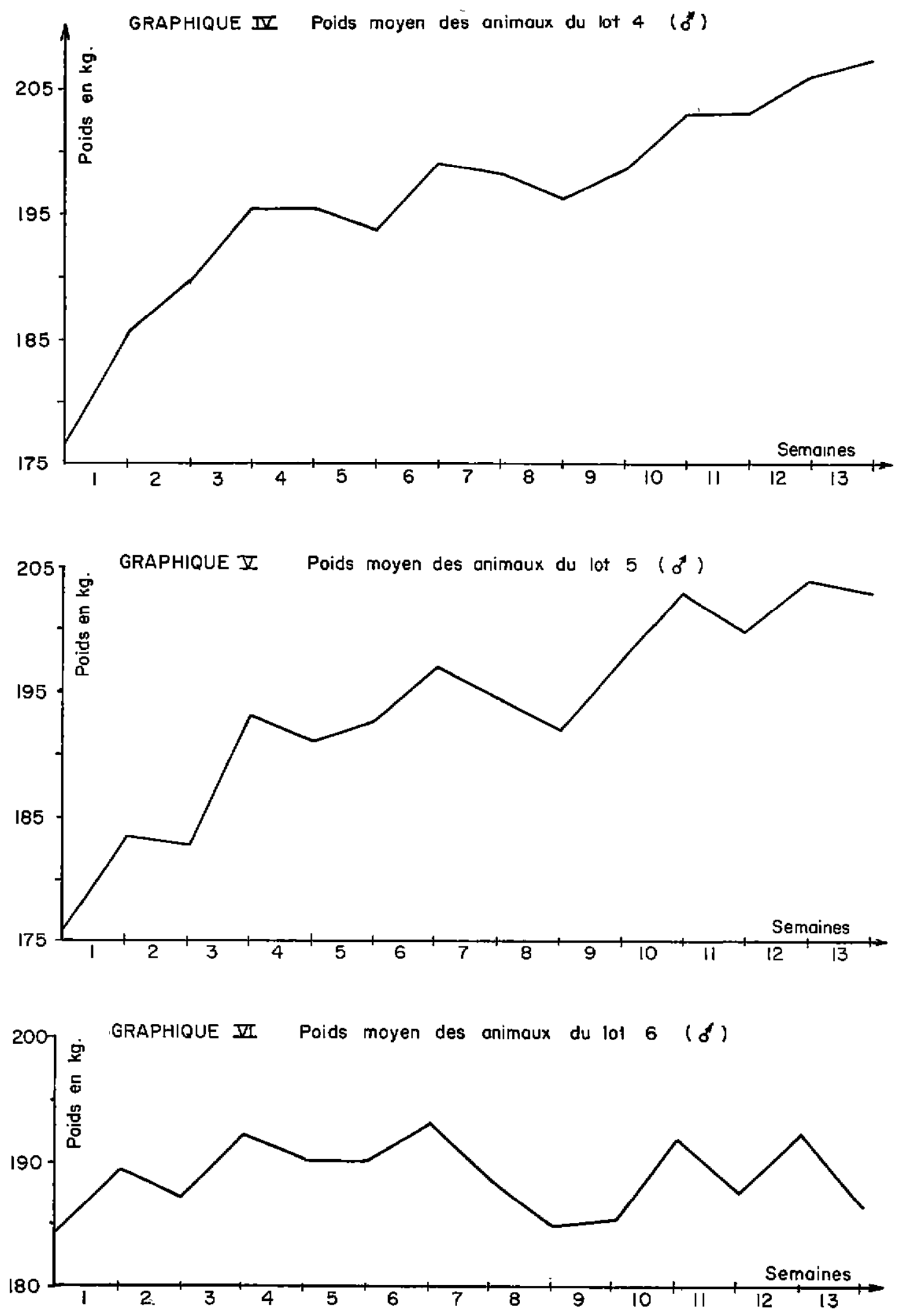
TABLEAU $V_{1}$ - Unilés Fourragères et Matières Azotées brutes des rations

\begin{tabular}{|c|c|c|c|c|c|c|c|c|}
\hline \multirow{2}{*}{ Lots } & \multicolumn{3}{|c|}{ Unitēs fourragères } & \multicolumn{4}{|c|}{ Matières azotées brutes (Kg) } & \multirow[t]{2}{*}{$\frac{M \cdot A \cdot B .}{U \cdot F \cdot}$} \\
\hline & Foin & Manioc & Total & Urée & Foin & Manioc & Total & \\
\hline I & 2380 & I89 & 2569 & 0 & $3 I 5,5$ & 4,6 & 320 , I & 124,6 \\
\hline 2 & $28 \mathrm{I} 4$ & I89 & 3003 & I 3 I & 373 & 4,6 & 508,6 & 169,3 \\
\hline 3 & 2582 & I096 & 3678 & I 31 & 342 & 26,8 & 499,8 & I35, 8 \\
\hline 4 & 2937 & 189 & $3 \mathrm{I} 2 \mathrm{I}$ & I 3 I & 389,2 & 4,6 & 524,8 & I $68, I$ \\
\hline 5 & 2390 & 1096 & 3486 & I 3 I & 316,7 & 26,8 & 474,5 & I36, I \\
\hline 6 & $2 I 3 I$ & 1096 & 3227 & 0 & 282,4 & 26,8 & 309,2 & 95,8 \\
\hline
\end{tabular}

Pour les lots 3 et 5 ce rapport est d'environ $136 \mathrm{~g}$ par U. F. ce qui ne satısfait pas totalement les besoins d'entretien et de croissance ; avec moins d'U. F., les lots 2 et 4 (manioc $250 \mathrm{~g} / \mathrm{jour}$ ) ont obtenu un gain de poids supérieur justifié par l'apport de $168 \mathrm{~g}$ de matières azotées brutes par $U$. F., ce qui se rapproche des normes pour des animaux de 1 an. Ces lots 2 et 4 sont ceux qui ont mangé le plus de foin.

Les lots 1 et 6 qui n'ont pas bénéficié d'urée ont nettement moins pris de poids. La distribution complémentaire de manioc au lot 6 a un effet néfaste car elle contribue d̀ diminuer la consommation de foin, et par conséquent elle abaisse le ropport $M$. A. B./U. F. qui de $87,2 \mathrm{~g}$ pour le lot 1 passe à $71 \mathrm{~g}$ pour le lot 6 .

Cela nous rappelle que le manioc est un aliment concentré très déséquilibré, mais très énergétique bien que très pauvre en protides, en minéraux et en vitamines.

Le foin, même médiocre, est bien mieux doté.

Pour de jeunes animaux, qui ont un besoin azoté important, le manioc ne peut être un appoint énergétique valable qu'en quantité limitée, et sous réserve d'une complémentation adéquate.

Le problème peut se poser de la même manière pour des bceufs à l'embouche, mais en termes plus nuancés car les besoins azotés sont alors moins importants.

Si l'on se pose le problème de la transformation de l'énergie de la ration, on constate qu'avec les lots 2 et 4 des résultats convenables, eu égard à l'encombrement des rations, ont pu être obtenus.

Avec le lot 2 (femelles) le gain de poids moyen journalier de $300 \mathrm{~g}$ par animal est obtenu par la consommation de 3 U. F. par jour. Cela donne le kilo de crồt pour $10 \mathrm{U}$. F. en moyenne pour l'ensemble du troupeau considéré.

Avec les mâles (lot 4) la croissance journalière a été de $330 \mathrm{~g}$ par animal, pour une consommation de 3,10 U. F. Le kilo de croît est ici obtenu avec 9,40 U. F.

Les résultats sont homogènes, et permettent d'envisager le problème sous l'angle économique.

C'est ce que nous allons explıciter.

\section{3o Bilan économique.}

Le coût du kilogramme de polds gagné par les animaux des divers lots peut être calculé car nous disposons du prix de tous les éléments.

Il est le suivant exprimé en francs malgaches (F.M.G.) :

\begin{tabular}{|c|c|}
\hline Foin (le kg) & \\
\hline Manioc sec (le kg) & \\
\hline Urée (le $\mathrm{kg}$ ) ..... & \\
\hline Farine d'os (le kg) & \\
\hline Sel $($ le $k g) \ldots \ldots$ & \\
\hline Vitamine $A(1.000 .0$ & 00 U. I.) \\
\hline
\end{tabular}

Un ouvrier agricole a été employé à plein temps pendant 3 mois pour assurer les distributions d'aliments aux 6 lots d'animaux. 
L'amortissement des clôtures et des râteliers a.été néglıgé.

L'intervention des chercheurs pour l'établissement du protocole expérimental, sa mise en place, ef l'exploitation des résultats n'entre pas en ligne de compte.

En fonction des quantités consommées, qui sont indiquées au tableau II et au tableau IV, compte tenu des prix indiqués ci-dessus et des gains de poids par lot (Tableau III), on peut dresser le bilan suivant: (Tableau VI).

L'examen final des prix de revient des kilos gagnés, confirme que l'alimentation du lot 6 conduit à un échec total sur le plan économique.

TABLEAU VI

Prix de revient du $\mathrm{Kg}$. de poids vif gagné (exprimé en Francs Malgaches)

\begin{tabular}{|c|c|c|c|c|c|c|}
\hline $\mathrm{LOTS}$ & I & 2 & 3 & 4 & 5 & 6 \\
\hline Foin & II.904 & I4.072 & $\mathrm{I} 2.9 \mathrm{I} 2$ & I 4.688 & II. 952 & 10.656 \\
\hline Manioc & 2.100 & 2.100 & 12.180 & 2. 100 & I2. I80 & I2. I80 \\
\hline Urëe & 0 & I. 739 & I. 739 & I. 739 & 1.739 & 0 \\
\hline Os & 600 & 600 & 600 & 600 & 600 & 600 \\
\hline Sel & 250 & 250 & 250 & 250 & 250 & 250 \\
\hline Vitamine A & I60 & $I 60$ & I60 & I60 & I60 & I60 \\
\hline Main d'Oeuvre & 3.000 & 3.000 & 3.000 & 3.000 & 3.000 & 3.000 \\
\hline TOTAL & I8.014 & $2 I .92 I$ & $30.84 \mathrm{I}$ & 22.537 & 29.881 & 26.846 \\
\hline Gain de poids $(\mathrm{Kg})$ & 85,8 & 302,5 & 245,3 & 332,2 & 283,8 & I 4,3 \\
\hline $\begin{array}{l}\text { Prix de Revient } \\
\text { du Kilo }\end{array}$ & 2 IO & 72 & I26 & 67,5 & IOS & I. 877 \\
\hline
\end{tabular}

On remarque que les lots ayant reçu de l'urée ont une rentabilité meilleure que le lot 1 qui n'en a pas reçu : parmi eux, les lots 2 et 4 n'ayant eu que $250 \mathrm{~g}$ de manioc par animal et por jour ont donné le prix de revient le plus bas.

L'urée, dans ce cas le plus propice, permet d'obtenir un prix de revient au kilo de croît qui n'est que le tiers de celui obtenu sans urée dans les mêmes conditions. On conviendra qu'ıl y a là un résultat spectaculaire.

$\mathrm{Si}$ au cours de cette période critique du sevrage on parvient à faire $1 \mathrm{~kg}$ de croît pour $70 \mathrm{~F}$. M. G., on réalise un progrès qui nous rapproche du but final : la production économique de viande de bœuf.

\section{CONCLUSION}

L'effet favorable de l'urée dans une alimentation des veaux à base de foin s'est vu entièrement confirmé. Elle a permis une consommation plus importante de foin, et une melleure utilisation digestive, qui s'est manifestée par des gains de poids plus que triplés.

L'apport d'amidon par du manioc doit rester modéré, car une forte quantité déséquilıbre la ration, en ce qui concerne les matières azotées surtout. L'alımentation devient plus onéreuse, pour des gains de poids plus faibles, et la rentabılité dıminue fortement.

Dans les conditions d'alimentation que nous avons déterminées comme les plus favorables, les animaux, malgré la saison qui est la plus mauvaise, malgré le «stress » du sevrage, malgré l'encombrement exagéré de leur ration, ont pu prendre plus de $300 \mathrm{~g}$ par jour. C'est un gage de bon démarrage dès la fin de «l'épreuve», car ils pourront profiter au mieux de l'herbe de saison des pluies. 


\section{SUMMARY}

The use of urea in the diel of weaning calves

In Madagascar, at Kianjasan Research Centre, 66 young cattle ( $3 / 4$ brahma $x$ $1 / 4$ malagasy zebu) have been fed during their weaning with a diet of hay, ad libitum, to which urea was added.

The consequences on the weight gains as well as nutritional and economic results are recorded and discussed.

The favourable effect of urea in the hay diet of calves has been completely confirmed.

\section{RES UMEN}

Utilización de la urea para la alimentación de los ferneros durante el destete

En Madagascar, en el centro de Kianjasoa, se alımentaron 66 terneros (3/4 brahma $\times 1 / 4$ cebú de Madagascar) durante su destete con una ración principaimente constituida por heno $y$ por urea como aditivo.

Se notan y se discuten los resultados ponderales así como los balances alimentıcios y económicos.

Se confirma enteramente la acción favorable de la urea adicionada con el heno en la alimentación de los terneros.

\section{BIBLIOGRAPHIE}

1. BUCK (G.) et Coll. - Effets de l'urée et de la vitamine $A$ sur les jeunes métis brahmanzébu malgache sevrés pendant la saison sèche dans la zone sédimentaire de l'ouest malgache. Bull. Madagoscor, 1965, 15 (224), 65-72 (cette publication contient des erreurs et omissions imputables à l'édıteur).

2. FERRANDO (R.). - L'urée dans l'alimentation des ruminants. Rev. Méd. vét., 1956, 107, 10-21, 96-108.

3. HART (E. B.) ef Coll. - The utilization of simple nitrogenous compounds such as urea and ammonium bicarbonate by gro- wing calves. J. dairy Sci., 1939, 22 (10), 785-98.

4. JARRIGE (R.). - L'utilisation des glucides alimentaires par les ruminants. Ann. Nutr. Alim., 1953, 7, 245-302, 339-406.

5. LADRAT (J.). - L'urée aliment. Rev. Méd. vét., 1954, 105, 287-92.

6. LOOSLI (J.K.) et Coll. - Synthesis of amino acids in the rumen. Science, 1949, 110, 144-45.

7. WATSON (C. J.) el Coll. - Scl. Agri., 1949, $29,173-188$. 